\title{
IN VITRO INVESTIGATION OF THE EFFECT OF A DYSFUNCTIONAL BILEAFLET MECHANICAL AORTIC VALVE ON FLOW CHARACTERISTICS IN THE ASCENDING AORTA
}

\author{
Ahmed Darwish $^{1 *}$, Wael Saleh ${ }^{1,2}$, Giuseppe Di Labbio ${ }^{l}$, Lyes Kadem ${ }^{1}$ \\ ${ }^{1}$ Mechanical, Industrial, and Aerospace Engineering Department \\ Concordia University \\ Montreal, QC, Canada \\ *email: 1cfd@encs.concordia.ca \\ ${ }^{2}$ Mechanical Engineering Department \\ Assiut University, Egypt
}

\begin{abstract}
Heart valve replacement is still the optimal solution in young patients with severe symptomatic heart valve disease. In those patients, bileaflet mechanical heart valves (BMHV) are preferred to biological valves because of their higher durability. However, thrombus formation has been reported as a common complication following BMHV implantation despite a permanent anticoagulation therapy. Thrombus formation can lead to valve leaflet dysfunction, a lifethreatening event that requires immediate surgical intervention. This study aims to investigate the fluid dynamics downstream of a dysfunctional bileaflet mechanical aortic valve. A bileaflet ON-X mechanical aortic valve is used with different dysfunctional configurations. In this study, partially and totally blocked configurations of one of the leaflets is investigated relative to its orientation with the sinus of Valsalva. Time-resolved two-dimensional particle image velocimetry measurements are performed to investigate flow field characteristics in the ascending aorta in terms of velocity and vorticity fields and circulation. Vorticity and circulation in the aorta are significantly affected by the valve orientation with respect to the sinus of Valsalva.
\end{abstract}

\section{Bileaflet Mechanical Heart Valve; Particle Image Velocimetry; Hemodynamics.}

\section{INTRODUCTION}

Heart valve failure is a known cardiovascular disease where valves fail to open or close properly. A report from the American Heart Association mentioned that the prevalence of heart valve diseases in the United States is $2.5 \%$ in 2013[1].

Treatment of valvular diseases ranges from adopting healthy lifestyle up to valve replacement [2] which is recommended for severe symptomatic cases.

Bileaflet Mechanical Heart Valve (BMHV) is a preferable choice for valve replacement in young patients because of its reported longer durability compared to prosthetic biological heart valves (BHV) [3], [4]. However, BMHV can be affected by thrombus of pannus formation [5]-[7]. Thrombosis formation has been shown to be related to non-physiological flow characteristics at the vicinity of the valve leaflets [5], where the shear stress levels may cause blood cell damage that would lead to platelet activation and clot formation [5], [8], [9]. Thrombus formation is more anticipated in BMHV than in BHV [6]. The incidence rate of prosthetic valve thrombus ranges between $0.03 \%$ - $5.7 \%$ per year [7]. Moreover, its occurrence is as high as $24 \%$ within the first year of the valve replacement [7]. This highly anticipated thrombus formation is expected to cause a dysfunction of one or both BMHV leaflets which will inevitably affect the fluid dynamics and hemodynamics in the ascending aorta [5].

Few previous studies investigated the flow past a dysfunctional BMHV. They considered the fluid dynamics and hemodynamics changes induced by the dysfunctional valve. In addition, they investigated the ability of current diagnostic tools to detect BMHV dysfunction.

Smadi et al. [10] first investigated numerically the steady flow through a defective BMHV at different flow and pathological conditions. They reported that Doppler echocardiography assessment of the dysfunctional valve is suboptimal mostly in the evaluation of transvalvular pressure gradients. The same authors [11] further investigated experimentally and in vivo the flow downstream of a dysfunctional BMHV. They showed that current clinical guidelines cannot accurately detect the presence of a dysfunctional BMHV and suggested new clinical parameters. Finally, Shahriari et al. [12] investigated numerically the shear stress accumulation on blood components in a dysfunctional BMHV by using smoothed particle hemodynamics. Their study was the first using a fully mesh-free method to cardiovascular flows. They reported that a dysfunctional BMHV leads to significantly more damage to blood components compared to a normal valve. 
In the previous studies, the orientation of the dysfunctional leaflet with respect to the sinus of Valsalva (SV) was not investigated. Despite the fact that bileaflet valves are usually installed with one leaflet facing one sinus and the other leaflet being shared by the other two sinuses [13]-[16]. This is an important issue since the development of the sinus vortex (washout vortex) and the coronary flow are both linked to the leaflet position relative to the sinus [5], [17]-[19]. Therefore, it is important to investigate the direct impact of BMHV dysfunction on flow characteristics downstream of the valve but also in the sinus region. This study aims to investigate experimentally using time-resolved two-dimensional (2D) particle image velocimetry the effect of a dysfunctional BMHV on flow characteristics in the ascending aorta.

\section{METHODOLOGY}

\section{A. Silicone molding}

An anatomically accurate aorta, left atrium and a simplified symmetric left ventricle are constructed using silicone molding. The molding process is done by adding a silicone rubber base to a curing catalyst (XIAMETER RTV-4234-T4 Base and XIAMETER T4/T4 O Curing Agent), with a ratio of 10:1 by mass respectively. The cured silicone has a Young modulus of 1.675 $\mathrm{MPa}$. The silicone is coated on a three-dimensional printed core mold of the parts. Four layers are coated, then, the parts are mounted to a rotating arrangement (speed of rotation $=10 \mathrm{rpm}$ ) to ensure a homogenous distribution of silicone over the core mold surface. The final thickness of the silicone layers ranges between $2-3 \mathrm{~mm}$.

\section{B. In vitro left heart simulator}

The left heart is assembled and mounted to a custom-made double activation in vitro heart simulator which is shown in Fig.1. The heart simulator has an activation box where the left ventricle is placed. The activation box is made of plexiglass (with index of reflection $=1.49$ ) which is filled with blood mimicking fluid and is connected to a cylinder which contains a compliance chamber to adjust the left ventricle compliance. A piston is sliding inside the cylinder to transfer the stroke from a linear motor to the left ventricle. Above the activation box, both aorta and left atrium are connected to the left ventricle. The aorta is placed inside a plexiglass box and is connected to the top of the open reservoir. The four pulmonary veins branching from the left atrium are all connected to the bottom of the reservoir. On the system, the left atrium is also activated by using a cam-follower arrangement mounted on a servomotor which transfers its motion to the left atrium. Both linear and servomotor are controlled with custom-made LabView interface which controls the timing and stroke of both motors. The liquid used is a mixture of water/glycerol with a volumetric ratio of $60 \% / 40 \%$, respectively. The mixture has an index of reflection $=1.4$ at $20^{\circ} \mathrm{C}$, while its dynamics viscosity is $=4 \mathrm{cP}$ at $23^{\circ} \mathrm{C}$ and its density is $=1080 \mathrm{~kg} / \mathrm{m}^{3}$. The flow rate is recorded for each case by using a magnetic inductive flow sensor with a flow range from 0.03-6.6 g/min and with a resolution of $0.01 \mathrm{~g} / \mathrm{min}$ (Prosense FMM50-102, Germany), while the pressure is recorded by using a fiber optic pressure sensor with a pressure range up to $300 \mathrm{mmHg}$ and with a resolution of $<0.3 \mathrm{mmHg}$ (FISO FOP-M260, Canada).

\section{Mechanical aortic valve}

The mechanical aortic valve used in this study is a bileaflet mechanical heart valve (On-XA-25, (manufactured by CryoLife, Inc, GA, USA)) with an inside diameter of $24.9 \mathrm{~mm}$. The valve is assembled and placed inside the aorta model. The leaflets orientation relative to the sinuses of Valsalva is selected based on previous studies [13]-[16].

The investigated dysfunctional cases are summarized in Table 1. In order to induce partial valve leaflet dysfunction, a plastic wire of $0.7 \mathrm{~mm}$ diameter is placed at accurately

calculated positions relative to the leaflets to work as an obstacle to leaflet opening. The level of leaflet dysfunction is confirmed by analyzing the opening angle of each leaflet and comparing it to the reported values by the manufacturer. The small sized plastic wire has the advantage of not interfering with the flow upstream of the valves. Complete valve leaflet dysfunction cases (C and D) are achieved by gluing the leaflet with the valve's inner casing.

\section{Particle image velocimetry measurements}

Time-resolved 2D Particle image velocimetry (PIV) measurements are carried out using a YLF laser with a $10 \mathrm{~mJ}$ output energy at $1 \mathrm{kHz}$ at $527 \mathrm{~nm}$ wave length (Litron Laser, UK). The laser sheet is placed as shown in Fig. 2. Images are captured using a Phantom V9.1 high speed camera with 1000 fps at a full resolution of $1632 \times 1200$ pixels (Vision Research, Inc., USA). Both laser and camera are controlled using a highspeed controller (Lavision $\mathrm{GmbH}$, Germany). The number of recorded frames is 400 and the recording duration is $1 \mathrm{~s}$. The fluid inside the heart simulator is seeded with polyamide seeding particles with a mean particle diameter of $50 \mu \mathrm{m}$ and an index of reflection of 1.5 (Dantec Dynamics A/S, Denmark).

Each captured frame contains two pairs of images where the time interval $\Delta \mathrm{t}$ between the two pairs is $600 \mu$ s for cases $\mathrm{N}$, $\mathrm{B}$, and $\mathrm{C}$, while $\Delta \mathrm{t}$ is $400 \mu \mathrm{s}$ for $\mathrm{A}, \mathrm{D}$, and $\mathrm{E}$.

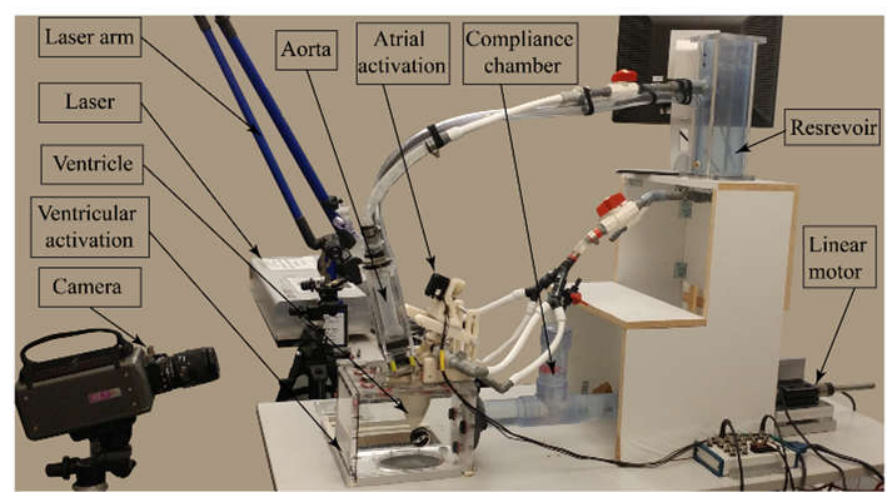

Figure 1 Experimental setup showing the camera, the laser, and the custommade heart simulator components. 


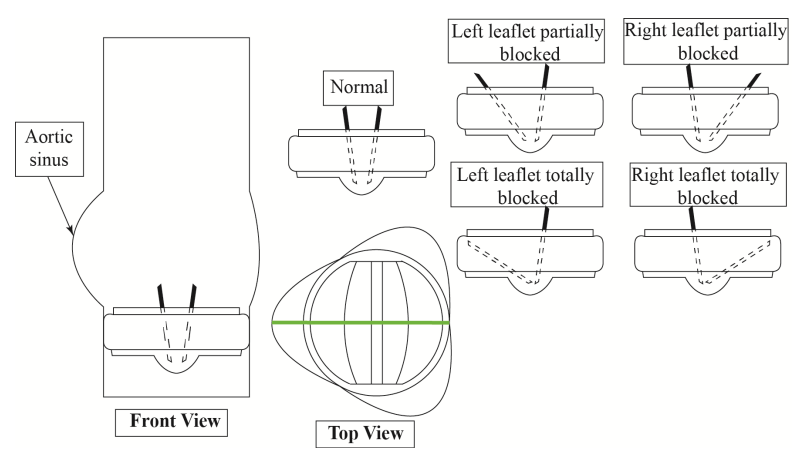

Figure 2 Front and top views of aorta showing the position of the aortic valve and the leaflets orientation with respect to the sinuses of Valsalva, beside the different dysfunction configurations of valve's leaflets. In the top view, green line represents the particle image velocimetry measurement plane.

TABLE 1. SUMMARY OF INVESTIGATED CASES AND MAXIMAL VELOCITY RECORED AT THE PEAK OF SYSTOLE

\begin{tabular}{|c|c|c|c|c|}
\hline Symbol & Case & $\begin{array}{l}\text { Dysfunction } \\
(\%)\end{array}$ & $\begin{array}{l}\text { Maximum } \\
\text { flow } \\
\text { velocity } \\
(\mathrm{m} / \mathrm{s})\end{array}$ & $\begin{array}{l}\text { Percentage of } \\
\text { velocity } \\
\text { increase relative } \\
\text { to normal } \\
\text { operation (\%) }\end{array}$ \\
\hline $\mathbf{N}$ & Normal & Null & 1.26 & Null \\
\hline $\mathbf{A}$ & $\begin{array}{l}\text { Left leaflet } \\
\text { partially } \\
\text { dysfunctional }\end{array}$ & $40-45 \%$ & 1.41 & $11.9 \%$ \\
\hline B & $\begin{array}{l}\text { Right leaflet } \\
\text { partially } \\
\text { dysfunctional }\end{array}$ & $40-45 \%$ & 1.39 & $10.3 \%$ \\
\hline C & $\begin{array}{l}\text { Left leaflet } \\
\text { totally } \\
\text { dysfunctional }\end{array}$ & $100 \%$ & 2.37 & $88.8 \%$ \\
\hline D & $\begin{array}{l}\text { Left leaf leaflet } \\
\text { totally } \\
\text { dysfunctional }\end{array}$ & $100 \%$ & 2.48 & $96.8 \%$ \\
\hline
\end{tabular}

The difference in the time interval between the investigated cases is due to the difference in the expected maximum flow velocity and to fulfill the requirements of the "1/4th law"

DaVis 7.2 software (Lavision GmbH, Germany) is used to post-process the recorded images where it calculates the velocity vectors from the raw images by using a fast Fourier transform cross-correlation with an initial 64x64 squared interrogation window (with three passes) with $50 \%$ overlap down to a $32 \times 32$ circular window (with two passes) with $50 \%$ overlap. A linear filter is then being applied to filter the noisy vectors.

A trigger is used between the heart simulator and DaVis7.2 so that each recording starts at the same time during the cardiac cycle (start of left ventricle systole). The recording is taken after 20 cycles lapse to ensure that the flow has reached its normal operation conditions.

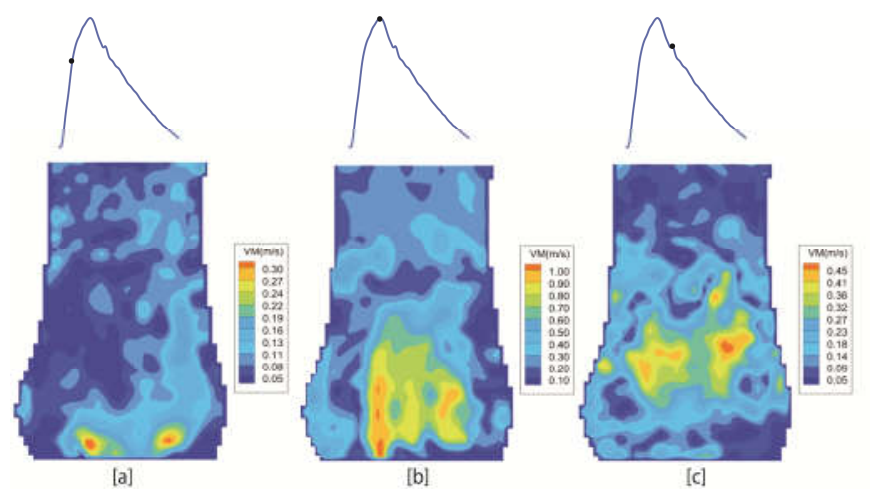

Figure 3 Velocity contours for normal case at different instants, [a] when the leaflets open, [b] at the peak of systole, [c] when the leaflets close.

\section{RESULTS}

\section{A. Velocity field downstream of the BMHV}

The maximal velocity at the peak of systole is summarized for all cases in TABLE 1. Figure 3 shows the velocity contours at the measurement plane at different phases during the cardiac cycle for the normal case. The normal maximum velocity for the valve is consistent with the reported values in [8], [17] by taking into consideration that the mean flow rate in this study is $3.8 \mathrm{l} / \mathrm{min}$. The velocities are much higher for the totally blocked cases which is directly related to the reduction in the effective orifice area (EOA) of the valve. The velocities obtained for cases $\mathrm{C}$ and $\mathrm{D}$ also agree with the values reported by [2].

\section{B. Vorticity and circulation}

To evaluate the effect of valve dysfunction on the flow field in the ascending aorta, vorticity contours are shown in Fig. 4 for each case at the peak of systole. The vorticity is calculated using (1), using a $4^{\text {th }}$ order compact Richardson scheme:

$$
\omega_{\mathrm{z}}=0.5(\delta v / \delta x-\delta u / \mathrm{d} y)
$$

where $\omega_{z}$ is the vorticity in z plane, $\delta v / \delta x$ is the derivative of $\mathrm{y}$-velocity component in $\mathrm{x}$ direction, and $\delta u / \mathrm{d} y$ is the derivative of $\mathrm{x}$-velocity component in $\mathrm{y}$ direction.

In the normal case, vorticity values in both directions are almost the same despite a higher value at the left side.

For a dysfunctional left leaflet or totally blocked (cases A, C), higher negative vorticity values are noticed, however the positive vorticity (counter clockwise rotation) dominates the measurements plane as shown in Fig. 4 a, b. The opposite behavior is noticed when the right leaflet is blocked, where clockwise rotation (negative sign vorticity) dominated the flow field as shown in Fig. 4 c, d. Therefore, the magnitude and distribution of circulation regions inside aorta is dependent on the position of the blocked leaflet. 


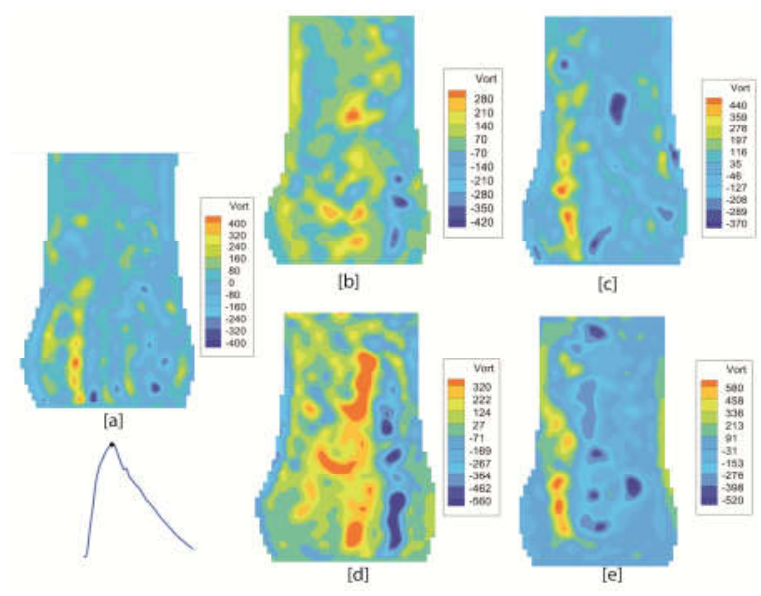

Figure 4 Vorticity contours (1/s) at the peak systole for [a] normal case, [b]left leaflet partially blocked, [c] right leaflet partially blocked, [d] left leaflet totally blocked, and [e] right leaflet totally blocked

Circulation, $\Gamma$ is calculated by integrating the vorticity over the $2 \mathrm{D}$ area in the sinus of Valsalva (SV) using (2) and is plotted against time for all cases as shown in Figure 5.

$$
\Gamma=\iint_{\mathrm{A}(\mathrm{x}, \mathrm{y})} \omega_{\mathrm{z}} d x d y
$$

As shown in Fig.5, circulation magnitude and direction inside the SV is dependent on the position of the blocked leaflet relative to the SV. In the normal case, the circulation inside the SV is positive during systole. When the right leaflet is blocked, positive circulation is also noticed in both $\mathrm{B}$ and $\mathrm{D}$, however at A when the left leaflet is partially blocked, we still can notice a positive circulation which is of a lower value compared to the normal case $\mathrm{N}$. Case $\mathrm{C}$ caused a negative circulation inside the SV, which is also noticed to be lower in magnitude compared to case $\mathrm{D}$.

The effect of circulation inside the SV has been reported to be related to coronary flow and to wall shear stresses by [18], [19]. Cao and Sucosky [19] described the interaction between the circulation inside SV and the coronary flow. While Stein et al.[18] noticed that the function of SV is to reduce turbulence near the entry of the coronary artery. Therefore, future studies have to investigate the impact of BMHV dysfunction on coronary flow circulation.

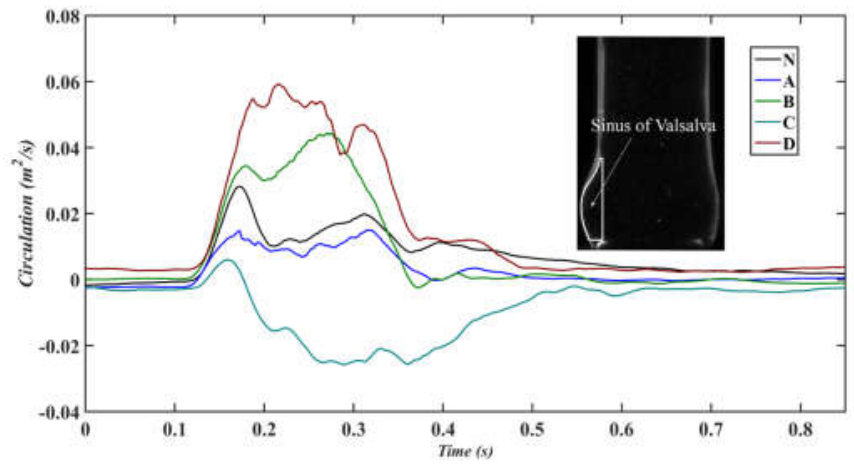

Figure 5 Temporal evolution of circulation -for (N) Normal case, (A) left leaflet partially blocked, (B) right leaflet partially blocked, (C) left leaflet totally blocked, and (D) right leaflet totally blocked-over the $2 D$ area of the sinus of Valsalva (in white).

\section{LIMITATIONS}

In this study, time-resolved 2D PIV measurements are performed while the flow pattern is expected to be of a 3D nature. Time-resolved 3D PIV measurement is one of our recommendations for future studies. Another limitation is using only one type of BMHVs. Future studies have to consider testing other types of valves and different valve sizes.

\section{CONCLUSION}

In this study we investigated the fluid dynamics downstream of a dysfunctional bileaflet mechanical aortic valve. An ON-X BMHV is used with different dysfunctional configurations. Both partially and totally blocked leaflet configurations are investigated relative to the orientation with the sinus of Valsalva. Time-resolved 2D particle image velocimetry measurements are performed to determine the flow field characteristics in the ascending aorta. Vorticity and circulation inside the aorta is significantly affected by the valve orientation with respect to the SV. The magnitude and direction of flow circulation inside the SV were also affected by the position of the dysfunctional leaflet relative to the SV. This observation should be considered in future studies dealing with the flow dynamics downstream of dysfunctional BMHVs. Further studies are required to fully understand the effect of a dysfunctional leaflet on flow topology and stability.

\section{REFERENCES}

[1] D. Mozaffarian et al., "Heart Disease and Stroke Statistics-2016 Update a Report From the American Heart Association," Circulation, vol. 133, no. 4, pp. e38-e48, 2015.

[2] R. A. Nishimura et al., "2014 AHA/ACC Guideline for the Management of Patients With Valvular Heart Disease: A Report of the American College of Cardiology/American Heart Association Task Force on Practice Guidelines," Circulation, vol. 129, no. 23, 2014.

[3] R. Bagur, P. Pibarot, and C. M. Otto, "Importance of the Valve Durability-Life Expectancy Ratio in Selection of a Prosthetic Aortic Valve," Heart, vol. 103, no. 22, pp. 1756-1759, 2017.

[4] Y. Misawa, A. Muraoka, K. Aizawa, and S. Ohki, "Recurrent Dysfunction after Open-Heart Valve Surgery," vol. 3, no. 2, pp. 3-5, 2016.

[5] L. P. Dasi, H. A. Simon, P. Sucosky, and A. P. Yoganathan, "Fluid Mechanics of Artificial Heart Valves," Clin. Exp. Pharmacol. Physiol., vol. 36, no. 2, pp. 225-237, 2009.

[6] R. Roudaut, K. Serri, and S. Lafitte, "Thrombosis of Prosthetic Heart Valves: Diagnosis and Therapeutic Considerations," Heart, vol. 93, no. 1, pp. 137-142, 2007.

[7] J. Salamon et al., "Mechanical Valve Obstruction: Review of Diagnostic and Treatment Strategies," World J. Cardiol., vol. 7, no. 12, pp. 875$881,2015$.

[8] F. Sotiropoulos, T. B. Le, and A. Gilmanov, "Fluid Mechanics of Heart Valves and Their Replacements," Annu. Rev. Fluid Mech., vol. 48, no. 1, pp. 259-283, 2016.

[9] A. P. Yoganathan, K. B. Chandran, and F. Sotiropoulos, "Flow in Prosthetic Heart Valves: State-of-the-Art and Future Directions," Ann. Biomed. Eng., vol. 33, no. 12 SPEC. ISS., pp. 1689-1694, 2005.

[10] O. Smadi, M. Fenech, I. Hassan, and L. Kadem, "Flow Through a Defective Mechanical Heart Valve: A Steady Flow Analysis," Med. Eng. Phys., vol. 31, no. 3, pp. 295-305, 2009.

[11] O. Smadi, J. Garcia, P. Pibarot, E. Gaillard, I. Hassan, and L. Kadem, "Accuracy of Doppler-Echocardiographic Parameters for the Detection of Aortic Bileaflet Mechanical Prosthetic Valve Dysfunction," Eur. Heart J. Cardiovasc. Imaging, vol. 15, no. 2, pp. 142-151, 2014. 
[12] S. Shahriari, H. Maleki, I. Hassan, and L. Kadem, "Evaluation of Shear Stress Accumulation on Blood Components in Normal and Dysfunctional Bileaflet Mechanical Heart Valves Using Smoothed Particle Hydrodynamics," J. Biomech., vol. 45, no. 15, pp. 2637-2644, 2012.

[13] I. Borazjani and F. Sotiropoulos, "The Effect of Implantation Orientation of a Bileaflet Mechanical Heart Valve on Kinematics and Hemodynamics in an Anatomic Aorta.," J. Biomech. Eng., vol. 132, no. 11, p. $111005,2010$.

[14] L. Haya and S. Tavoularis, "Effects of Bileaflet Mechanical Heart Valve Orientation on Fluid Stresses and Coronary Flow," J. Fluid Mech., vol. 806, pp. 129-164, 2016.

[15] P. Kleine, M. Scherer, U. Abdel-Rahman, A. A. Klesius, H. Ackermann, and A. Moritz, "Effect of Mechanical Aortic Valve Orientation on Coronary Artery Flow: Comparison of Tilting Disc Versus Bileaflet Prostheses in Pigs," J. Thorac. Cardiovasc. Surg., vol. 124, no. 5, pp.
925-932, 2002.

[16] J. Laas, P. Kleine, M. J. Hasenkam, and H. Nygaard, "Orientation of Tilting Disc and Bileaflet Aortic Valve Substitutes for Optimal Hemodynamics," Ann. Thorac. Surg., vol. 68, no. 3, pp. 1096-1099, 1999.

[17] A. P. Yoganathan, Z. He, and S. Casey Jones, "Fluid Mechanics of Heart Valves," Annu. Rev. Biomed. Eng., vol. 6, no. 1, pp. 331-362, 2004.

[18] P. D. Stein, E. F. Blick, S. K. Shields, and F. Matta, "Sinus of Valsalva: A Converging Nozzle that Contributes to Stable Flow in the Coronary Arteries," J. Anat., vol. 225, no. 1, pp. 94-97, 2014.

[19] K. Cao and P. Sucosky, "Aortic Valve Leaflet Wall Shear Stress Characterization Revisited: Impact of Coronary Flow," Comput. Methods Biomech. Biomed. Engin., vol. 20, no. 5, pp. 468-470, 2017.

[20] M. Raffel, C. E. Willert, S. T. Wereley, and J. Kompenhans, Particle Image Velocimetry, vol. 79, no. 1. Springer Berlin Heidelberg, 2007. 